\title{
DESEMPENHO PRODUTIVO DO AMENDOIM SUBMETIDO A DIFERENTES ADUBAÇÕES VIA SOLO E FOLIAR
}

Gabriela Duarte Monaco'; Gabriel Augusto da Silva Cirilo²; João Vitor Pires Junqueira Reis'; Lucas Martins de Azevedo Souza ${ }^{4}$; Fernando de Oliveira Turci Sandrini ${ }^{5}$; Mateus Vieira Malaquias ${ }^{6}$

\author{
${ }^{1}$ Graduando em Engenharia Agronômica, FCAV/UNESP, Jaboticabal, SP, gabrielamonaco2@gmail.com; \\ ${ }^{2}$ Graduando em Engenharia Agronômica, FCAV/UNESP, Jaboticabal, SP; ${ }^{3}$ Graduando em Engenharia \\ Agronômica, FCAV/UNESP, Jaboticabal, SP; ${ }^{4}$ Graduando em Engenharia Agronômica, FCAV/UNESP, \\ Jaboticabal, SP; ${ }^{5}$ Graduando em Engenharia Agronômica, FCAV/UNESP, Jaboticabal, SP; ${ }^{6}$ Graduando em \\ Engenharia Agronômica, FCAV/UNESP, Jaboticabal, SP.
}

RESUMO: A cultura do amendoim (Arachis hypogaea L.) tem ciclo de aproximadamente 130 dias, o que possibilita a rápida ciclagem de nutrientes e fixação de nitrogênio no solo. Entretanto, ainda existem poucos estudos sobre o comportamento agronômico a diante das maneiras de aplicação e períodos de adubação, seja ela via solo ou foliar. Visando obter mais informações quanto a isso, foi implantado na fazenda experimental da FCAV/UNESP um experimento com a cultivar IAC OL3, em delineamento em blocos casualizados com 5 repetições e 5 tratamentos. As avaliações foram realizadas nas duas linhas centrais. Os dados foram submetidos a análise de variância e as medias comparadas pelo teste Tukey a 5\% de probabilidade. Não houve diferenças significativas entre os tratamentos. Todavia, todos os tratamentos apresentaram produtividades superiores a esperada pelo cultivar.

Palavras-Chave: Arachis hypogaea L., adubação, produtividade, pulverização

\section{INTRODUÇÃO}

O amendoim (Arachis hypogaea L.) é uma das oleaginosas mais importantes para o consumo humano. Juntamente com o feijão e a soja, o grão se destaca pelo fornecimento de alimento proteico e energético, mas também como um dos principais produtores de óleo (MARTIM et al., 2009). Ocupa uma área de 145,1 mil hectares produzindo em média $3.441 \mathrm{~kg} \mathrm{ha}^{-1}$ (CONAB, 2018).

A cultura apresenta facilidade de desenvolvimento na maioria dos tipos de solo, mas apresenta melhor desempenho em solos bem drenados, férteis e de textura arenosa (BELTRÃO et al., 2011). Nesse sentido, a adubação e nutrição influenciam não somente no desenvolvimento das plantas, mas também nos aspectos quantitativos e qualitativos da cultura (BOLONHEZI et al., 2005). Estudos relatando a desordem nutricional do amendoim mostraram que a omissão dos macronutrientes proporcionou a diminuição de crescimento e massa seca da parte área, raízes e a planta inteira, além da redução significativa na altura, no diâmetro do caule e no número de folhas (CORREIA, et al., 2011). 
16 e 17 de agosto de 2018, centro de convenções da FCAV/UNESP - Câmpus de Jaboticabal, SP

Contudo, os resultados obtidos no fornecimento nutricional do amendoim ainda não são concretos. Apesar da capacidade de extração dos nutrientes essenciais através das raízes, dos ginóforos e dos frutos em desenvolvimento, as respostas da cultura a diferentes fontes possuem variações (NAKAGAWA et al.,1993; MARUBAYASHI et al., 1997; FREIRE et al., 2007).

Neste contexto, o objetivo deste trabalho foi avaliar o desempenho agronômico da cultura do amendoim submetida a diferentes manejos no fornecimento dos nutrientes por meio da adubação no sulco de plantio juntamente com a aplicação foliar durante o desenvolvimento da cultura.

\section{MATERIAL E MÉTODOS}

O experimento foi conduzido em condições de campo na área experimental da Universidade

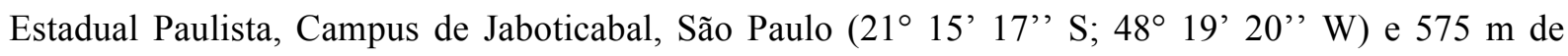
altitude, com classificação climática de Koeppen: Aw. O semeio do amendoim IAC OL3 - ciclo de 130 dias, foi realizado através de semeadora adubadora com dosador pneumático à vácuo em 09 de novembro de 2017. O experimento foi delineado em blocos casualizados com cinco tratamentos (tabela 1) e cinco repetições, com parcelas de 59,4 $\mathrm{m}^{2}$ (5,4x11 metros).

O controle fitossanitário foi realizado ao longo da condução do experimento com capinas manuais, aplicações de herbicida (glifosato), fungicida (piraclostrobina, fluxapiroxade, tetrachloroisophthalonitrile (clorotalonil), tebuconazol trifloxistrobina, ciproconazol e trifloxistrobina), inseticida (imidacloprido, beta-ciflutrina, deltametrina, lambda-cialotrina, tiametoxam e tiodicarbe) e acaricida (abamectina).

Tabela 1: Descrição dos tratamentos adotados no experimento

\begin{tabular}{|c|c|c|c|c|c|c|}
\hline Tratamento & $\begin{array}{r}\text { Tratamento } \\
\text { de sementes }\end{array}$ & Plantio & 20 DAP & 35 DAP & 60 DAP & 70 DAP \\
\hline 1 & $\begin{array}{c}\text { YaraVita } \\
\text { Raiz® }^{\circledR} \\
2 \mathrm{ml}^{\mathrm{kgg}}{ }^{-1}\end{array}$ & Absoluto ${ }^{\circledR}$ & $\begin{array}{c}\text { Amazinc }{ }^{\circledR} \\
0,5 \mathrm{~L} \cdot \mathrm{Ha}^{-1} \\
+ \text { Biotrac }^{\circledR} \\
\\
0,3 \mathrm{~L} \cdot \mathrm{Ha}^{1}{ }^{1}\end{array}$ & $\begin{array}{c}\text { Bortrac }{ }^{\circledR} \\
0,5 \text { L.Ha } \\
1 \\
(35 \text { e } 45 \\
\text { DAP) }\end{array}$ & $\begin{array}{c}\text { Thiotrac }{ }^{\circledR} \\
1 \text { L.Ha }^{-1}+ \\
\text { Biotrac } \AA \\
0,2 \text { L.Ha }{ }^{-1}\end{array}$ & $\begin{array}{l}\text { Folicare }{ }^{\circledR} \\
3 \mathrm{~kg} \cdot \mathrm{Ha}^{-1}\end{array}$ \\
\hline 2 & $\begin{array}{c}\text { YaraVita } \\
\text { Raiz }^{\circledR} \\
2 \mathrm{ml}^{-\mathrm{kg}^{-1}} \\
\end{array}$ & Absoluto ${ }^{\circledR}$ & $\begin{array}{c}\text { Biotrac }{ }^{\circledR} \\
0,3 \mathrm{~L} . \mathrm{Ha}\end{array}$ & & $\begin{array}{l}\text { Biotrac }{ }^{\circledR} \\
0,2 \text { L.há }^{-1}\end{array}$ & \\
\hline 3 & $\begin{array}{c}\text { YaraVita } \\
\text { Raiz®}^{\circledR} \\
2 \mathrm{ml}^{-\mathrm{kg}^{-1}} \\
\end{array}$ & Absoluto ${ }^{\circledR}$ & & & & $\begin{array}{l}\text { Folicare }{ }^{\circledR} \\
3 \mathrm{~kg} \cdot \mathrm{Ha}^{-1}\end{array}$ \\
\hline 4 & $\begin{array}{c}\text { YaraVita } \\
\text { Raiz®}^{\circledR} \\
2 \mathrm{ml}^{\circ} \mathrm{kg}^{-1} \\
\end{array}$ & $\begin{array}{l}\text { Adubação } \\
\text { convencional }\end{array}$ & & & & \\
\hline 5 & & $\begin{array}{c}\text { Adubação } \\
\text { convencional }\end{array}$ & & & & \\
\hline
\end{tabular}

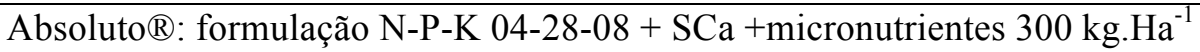

Amazinc ${ }^{\circledR}$ : Nitrogênio 1,9\%, Manganês 14\% e Zinco 19,5\% (p/p)

Biotrac ${ }^{\circledR}$ : Nitrogênio $5,6 \%$, Boro $1,1 \%$ e Zinco $1,1 \%(\mathrm{p} / \mathrm{p})$ 
16 e 17 de agosto de 2018, centro de convenções da FCAV/UNESP - Câmpus de Jaboticabal, SP

Bortrac ${ }^{\circledR}$ : Boro $10,9 \%(\mathrm{p} / \mathrm{p})$

Convencional: formulação N-P-K 04-30-10 $300 \mathrm{~kg} \cdot \mathrm{Ha}^{-1}$

Folicare ${ }^{\circledR}$ : Nitrogênio $12 \%$, Potássio 39\%, Magnésio 1,8\%, Enxofre 2,8\% (p/p)

Thiotrac $₫$ : Nitrogênio $15,2 \%$, Enxofre $22,8 \%$ (p/p)

YaraVita Raiz ${ }^{\circledR}$ : Nitrogênio 4\%, Carbono orgânico 6\%, Cobalto 1\%, Molibdênio 5\% (p/p)

Para aplicação dos adubos foliares foi utilizado um pulverizador costal pressurizado por $\mathrm{CO}^{2}$ com pressão constante de 1 bar, o espaçamento entre bicos na barra foi de $0,5 \mathrm{~m}$ e a altura de aplicação de $0,5 \mathrm{~m}$ em relação ao topo da cultura, utilizando ponta convencional tipo defletora (TT 110 02), com projeção vertical. A velocidade de deslocamento foi de $3,6 \mathrm{~km} \mathrm{~h}^{-1}$, resultando no volume de aplicação de $200 \mathrm{~L} \mathrm{ha}^{-1}$.

Foram analisadas a massa de grãos provenientes das duas linhas centrais, descartando as 4 linhas de bordadura. Os dados foram submetidos ao teste $\mathrm{F}$ da análise de variância (ANOVA) e quando significativo $(\mathrm{p}<0,05)$ as médias dos tratamentos foram comparadas entre si, utilizando-se o teste de Tukey à $5 \%$ de probabilidade, com auxílio do software de AgroEstat ${ }^{\circledR}$.

\section{RESULTADOS E DISCUSSÃO}

Considerando os diferentes tratamentos aplicados na cultivar de amendoim IAC OL3 foram analisados a produtividade e rendimento de grãos, de modo a estudar o melhor manejo nutricional para a cultura. $\mathrm{O}$ tratamento 1 apresentou uma produtividade média de 239,36 sacas/ha, e rendimento médio de grãos de 74,13\%. No tratamento 2 alcançou-se uma produtividade média de 239,30 sacas/ha e $74,96 \%$ de rendimento médio de grãos. Para o tratamento 3 foi obtido produtividade média de 248,52 sacas/ha e rendimento médio de grãos de $74,28 \%$. No tratamento 4 a produtividade média foi de 254,32 sacas/ha com rendimento médio de grãos de $76,16 \%$. A produtividade média do tratamento 5 foi de 233,24 sacas/ha e com rendimento médio de 73,78\% (tabelas 2 e 3).

Tabela 2. Produtividade da cultivar OL3 de amendoim submetida a diferentes manejos de adubação/ha.

Produtividades obtidas no experimento - sacas/ha

\begin{tabular}{|c|c|c|c|c|c|c|c|}
\hline Tratamentos & Bloco 1 & Bloco 2 & Bloco 3 & Bloco 4 & Bloco 5 & Média & \\
\hline 1 & 226,90 & 302,60 & 253,40 & 206,90 & 207,00 & 239,36 & A \\
\hline 2 & 210,10 & 245,00 & 247,10 & 268,80 & 225,50 & 239,30 & A \\
\hline 3 & 284,00 & 245,50 & 220,30 & 245,50 & 247,30 & 248,52 & A \\
\hline 4 & 240,50 & 215,40 & 285,20 & 254,10 & 276,40 & 254,32 & $\mathrm{~A}$ \\
\hline 5 & 224,10 & 245,20 & 243,00 & 259,20 & 194,70 & 233,24 & A \\
\hline
\end{tabular}

* Sacas de amendoim: 25 quilogramas equivalentes a grão em vagem

*Letras iguais não diferem significativamente pelo teste de Tukey a 5\% 
16 e 17 de agosto de 2018, centro de convenções da FCAV/UNESP - Câmpus de Jaboticabal, SP

Tabela 3. Rendimento de grãos em porcentagem

Rendimento de grãos - \%

\begin{tabular}{cccccccc}
\hline Tratamentos & Bloco 1 & Bloco 2 & Bloco 3 & Bloco 4 & Bloco 5 & Média & \\
\hline 1 & 74,03 & 77,24 & 76,84 & 68,81 & 73,75 & 74,13 & A \\
\hline 2 & 73,24 & 74,39 & 76,69 & 76,16 & 74,32 & 74,96 & A \\
\hline 3 & 76,97 & 75,88 & 76,10 & 68,37 & 74,07 & 74,28 & A \\
\hline 4 & 76,50 & 77,58 & 76,46 & 73,64 & 76,61 & 76,16 & A \\
\hline 5 & 77,20 & 66,35 & 72,25 & 75,38 & 77,72 & 73,78 & A \\
\hline
\end{tabular}

*Rendimento de grãos: relação entre peso de grãos e peso de grãos em vagem

*Letras iguais não diferem significativamente pelo teste de Tukey a 5\%

A resposta não significativa às adubações foliares pode-se presumir que seja devido a baixa resposta da cultura do amendoim a adubações diretas via foliar, sendo ela uma cultura mais responsiva a adubação residual de culturas anteriores, por via de resíduos orgânicos. (COPE et al.,1984; COX et al.,1982). É uma cultura que tem seu nível de produção mais impactado pela fertilidade do solo do que pela adubação, exceto pela calagem e adubações fosfatadas (Lima et al., 2009), e com baixa resposta para o potássio na produção de vagens (Gargantini et al., 1958; Nakagawa et al., 1971a, Nakagawa et al.,1993; Marubayashi et al., 1997; Freire et al., 2007; Lima et al., 2009), portanto as adubações realizadas que continham concentrações variáveis desses nutrientes supracitados podem ter tido pouco efeito expressivo devido ao comportamento típico da cultura do amendoim. Deve-se considerar também que o solo da área onde foi feito a semeadura, foi realizado análise de solo, encontrando os seguintes resultados para a camada de 0 a $20 \mathrm{~cm}$ : P, 22 mg.dm ${ }^{-3}$; MO, 28 g.dm-3; pH CaCl2 5,7; S$\mathrm{SO}_{4}{ }^{2-}, 7 \mathrm{mg} \cdot \mathrm{dm}^{-3} ; \mathrm{Ca}^{2+}, 24 \mathrm{mmol}_{\mathrm{c}} \cdot \mathrm{dm}^{-3} ; \mathrm{Mg}^{2+}, 12 \mathrm{mmol}_{\mathrm{c}} \cdot \mathrm{dm}^{-3} ; \mathrm{K}^{+}, 5,6 \mathrm{mmol}_{\mathrm{c}} \cdot \mathrm{dm}^{-3}, \mathrm{H}+\mathrm{AL}, 20$ $\mathrm{mmol}_{\mathrm{c}} \cdot \mathrm{dm}^{-3}$; SB, 41,6 mmol $\cdot \mathrm{dm}^{-3}$; CTC, $61,8 \mathrm{mmol}_{\mathrm{c}} \cdot \mathrm{dm}^{-3} ; \mathrm{V}, 67 \%$; e na camada de 20 a 40, com saturação por alumínio de $1 \%$. Portanto, de acordo com a tabela 4 , possuía teores médios ou altos dos macronutrientes, $\mathrm{P}, \mathrm{S}, \mathrm{K}, \mathrm{Ca}$ e $\mathrm{Mg}$, e baixa saturação por alumínio, o que pode ter tornado obsoleto o uso de adubações nas dosagens realizadas.

Tabela 4. Classes de interpretação dos macronutrientes em solos.

\begin{tabular}{|c|c|c|c|c|c|}
\hline \multirow{3}{*}{ Interpretação } & \multirow{2}{*}{$\begin{array}{l}\text { P resina } \\
\text { (anuais) }\end{array}$} & \multirow[t]{2}{*}{$\mathrm{S}-\mathrm{SO}_{4}{ }^{2-}$} & \multirow[t]{2}{*}{$\mathrm{K}^{+}$} & \multirow[t]{2}{*}{$\mathrm{Ca}^{2+}$} & \multirow[t]{2}{*}{$\mathrm{Mg}^{2+}$} \\
\hline & & & & & \\
\hline & \multicolumn{2}{|c|}{------mg/dm3------- } & \multicolumn{3}{|c|}{--------mmolc/dm3---------- } \\
\hline Muito baixo & $0-6$ & & $0-0,7$ & & \\
\hline Baixo & $7-15$ & $0-4$ & $0,8-1,5$ & $0-3$ & $0-4$ \\
\hline Médio & $16-40$ & $5-10$ & $1,6-3,0$ & $3-7$ & $5-8$ \\
\hline Alto & $41-80$ & $>10$ & $3,1-6,0$ & $>7$ & $>8$ \\
\hline Muito alto & $>80$ & & $>6,0$ & & \\
\hline
\end{tabular}




\section{CONCLUSÃO}

$\mathrm{Na}$ análise estatística pelo teste de Tukey a $5 \%$ de probabilidade não houve diferença significativa entre os tratamentos tanto para produtividade quanto para rendimento de grãos.

\section{AGRADECIMENTOS}

Aos membros do grupo PET AGRO, aos funcionários da FEPE - Fazenda de Ensino, Pesquisa e Extensão, à UNESP/FCAV - Campus de Jaboticabal, e à empresa Yara Brasil S.A.

\section{REFERENCIAS BIBLIOGRÁFICAS}

BELTRÃO, N. E. M; SOUSA JUNIOR, S. P.; OLIVEIRA, M. I. P.; FIDELES FILHO, J.; SILVA, M. N. B. Ecofisiologia do amendoim (Arachis hypogaea L.). In: BELTRÃO, N. E. M.; OLIVEIRA, M. I. P. - Ecofisiologia das culturas algodão, amendoim, gergelim, mamona, pinhãomanso e sisal. Brasília, DF. Embrapa. 2011, p. 125-162.

BOLONHEZI, D.; GODOY, I. J.; SANTOS, R. C. Manejo Cultural do Amendoim. In: SANTOS, R. C. (Ed. Téc.). O agronegócio do amendoim no Brasil. Campina Grande: Embrapa Algodão; Brasília: Embrapa Informação Tecnológica, p. 193- 244, 2005.

CONAB - Companhia Nacional de Abastecimento. Acompanhamento da safra brasileira de grãos. Mensal, v.5 - Safra 2017/18, n.5 - Quinto Levantamento, Brasília: Companhia Nacional de Abastecimento. p. 1-140, fev. 2018. Disponível em: http://www.conab.gov.br Acesso em: 30 mai. 2018.

CORREIA, M. A. R.; PRADO, R. M.; ALMEIDA, T. B. F.; PUGA, A. P.; BARBOSA, J. C.; Avaliação da desordem nutricional de plantas de amendoim cultivadas em solução nutritiva suprimidas de macronutrientes. Scientia Agraria, Curitiba, v.13, n.1, p.21-28, 2012.

FREIRE, M. L. F.; BELTRÃO, N. E. M.; RAO, T.V.R.; MENEZES, H.E.A. Cultivo do amendoim submetido a diferentes níveis de adubação e condições edafoclimáticas no sudoeste de Goiás. Revista Brasileira de Ciências Agrárias, Recife, PE, v.2, n.3, p.193-199, 2007.

INSTITUTO AGRONÔMICO DE CAMPINAS. Cultivares de Amendoim. Disponível em: $<$ http://www.iac.sp.gov.br/areasdepesquisa/graos/amendoim.php>. Acesso em: 23 de junho de 2018.

MARTIM, A.; ASSUNÇÃO, H. F.; LIMA, T. M.; Ensaio de competição para avaliar o desempenho produtivo de quatro variedades de amendoim, no sudoeste de Goiás. In: CONGRESSO BRASILEIRO DE PLANTAS OLEAGINOSAS, ÓLEOS, GORDURAS E BIODIESEL, 6., 2009, Montes Claro,MG. Anais..., Monte Claros 2009. Editado em CD-ROM.

MARUBAYASHI, O. M.; ROSOLEM, C.A.; NAKAGAWA, J.; ZANOTTO, M. D. Adubação fosfatada, produção e qualidade de sementes de populações de amendoim. Pesquisa Agropecuária Brasileira, Brasília, v.32, n.9, 1997. 
16 e 17 de agosto de 2018, centro de convenções da FCAV/UNESP - Câmpus de Jaboticabal, SP

MESCHEDE, D.K.; BRACCINI, A.L.; BRACCINI, M.C.L.; SCAPIM, C.A.; SCHUAB, S.R.P. Rendimento, teor de proteínas nas sementes e características agronômicas das plantas de soja em resposta à adubação foliar e ao tratamento de sementes com molibdênio e cobalto. Acta Scientiarum Agronomy, Maringá, v.26, n.2, p. 139-145, 2004.

NAKAGAWA, J.; NAKAGAWA, J.; IMAIZUMI, I.; ROSSETTO, C.A.V. Efeitos de fontes de fósforo e da calagem na produção de amendoim. Pesquisa Agropecuária Brasileira, Brasília, v.28, n.4, p.421-431, 1993.

PRADO, R.M. 500 perguntas e respostas sobre nutrição de plantas. Jaboticabal: FCAV/Genplant, 2009.

TASSO JR, L. C. MARQUeS, M. O.; NOGUEIRA, G. A., L. A cultura do amendoim. 1. ed. Jaboticabal: UNESP, 2004. 218 p. 